\title{
Producing ultrashort, ultraintense plasma-based soft-x-ray laser pulses by high-harmonic seeding
}

\author{
D. S. Whittaker, ${ }^{1, *}$ M. Fajardo, ${ }^{1}$ Ph. Zeitoun, ${ }^{2}$ J. Gautier, ${ }^{2}$ E. Oliva, ${ }^{3}$ S. Sebban,${ }^{2}$ and P. Velarde ${ }^{3}$ \\ ${ }^{1}$ Instituto Superior Técnico. Pontugal \\ ${ }^{2}$ Laboratoire d'Optique Appliquée, ENSTA. CNRS. Ecole Polytechnique, Palaisean. France. \\ ${ }^{3}$ Universidad Politecnica de Madrid, Avente Abascal, Madrid. Spain
}

\begin{abstract}
Simulations show that intense plasma-amplified pulses of $100 \mathrm{fs}$ duration and below are feasible by seeding specifically tailored plasma with an ultrashort pulse of high harmonic radiation. Seeding overcomes gain narrowing by driving amplifying media into saturation earlier, and compensates for reduced gain resulting from boosting the lasing transition linewidth. We conclude that ultrahigh intensities (above $10^{16} \mathrm{~W} \mathrm{~cm}{ }^{-2}$ ) could be reached.
\end{abstract}

PACS number(s): $42.55 . \mathrm{Vc}, 41.60 . \mathrm{Cr}, 52.38 .-\mathrm{r} .52 .50 . \mathrm{Jm}$

\section{INTRODUCTION}

Recently, a new generation of light sources has come of age with the development of soft-x-ray free-electron lasers [1]. These sources deliver soft-x-ray radiation with intensities above $10^{16} \mathrm{~W} \mathrm{~cm}^{-2}$ in ultrashort pulses of less than $100 \mathrm{fs}$ duration [2], opening up new regimes of interaction between light and matter. Although their average pulse energy is limited typically to $10-50 \mu \mathrm{J}$ [2], their short pulse durations have made possible the demonstration of techniques such as single-shot diffraction imaging [3] and time-resolved holography [4]. Conventional soft-x-ray lasers (SXRLs), relying on population inversions from highly charged states in a plasma, store a greater amount of energy, resulting in pulses as energetic as $10 \mathrm{~mJ}$ [5]. The range of applications of SXRLs is limited, however, by their relatively long pulse durations (typically $100-200 \mathrm{ps}$ ) due to the narrow spectral width of the soft-x-ray laser line, though SXRL pulses as short as $\sim 2$ ps have been reported [6]. Here, the possibility of generating ultraintense (>10 $10^{16} \mathrm{~W} \mathrm{~cm}^{-2}$ ), ultrashort (sub-100-fs) pulses using the scaled-down architecture of plasma-based amplifiers is studied. The proposed scheme uses suitably engineered plasma to amplify an initially relatively intense, very large bandwidth ( $\Delta v / v \simeq 0.01, v$ being the laser frequency) Gaussian pulse of high harmonic $(\mathrm{HH})$ radiation - the "seed." Such a scheme has already resulted in saturated amplification of $\mathrm{HH}$ seeds using plasma amplifiers generated from gaseous [7] and solid [8] targets and, very recently by Wang et al. [9], in measured pulse durations of $\sim 1$ ps. In this article, we briefly revisit spectral lineshape theory and benchmark a model to predict linewidth with experimental results of Koch et al. [10]. Using this model, we investigate plasma conditions and laser parameters necessary to keep the seeded beam duration close to and below 100 ts after amplification.

To determine the duration of unseeded SXRL pulses produced with quasi-steady-state schemes [11], streaked measurements record emission over several gain lifetimes. For Fourier-limited cases-such as those obtained in transient pumping [12]—emission during approximately only one gain lifetime is amplified and there is a direct inverse relationship between pulse duration and spectral bandwidth. The dominant broadening mechanisms under conditions required for SXRL operation are generally Doppler and collisional. Doppler broadening is inhomogeneous and results in a Gaussian profile (assuming the emitting ions have a Maxwellian velocity distribution). Collisional broadening is homogeneous and has a Lorentzian profile in the impact approximation [13].

Inhomogeneous broadening, in the absence of significant homogeneous broadening, causes a rebroadening of the line profile after saturation. However, saturation rebroadening has never been observed experimentally in typical laser-produced plasmas of relevance to this work (i.e., in those with a reasonably high mean charge state and-hence-electron density) [14], but it has been observed to increase in noble gas laser transitions when the interatomic collision frequency is reduced $[14,15]$. This suggests that collisional redistribution of velocity acts to homogenize the otherwise inhomogeneous Doppler broadening, destroying the rebroadening mechanism.

Furthermore, even if the linewidth of the amplifying transition were to rebroaden to its original extent following saturation, to produce sub-100-fs pulses with conventional plasma gain media via Doppler broadening alone would require ion temperatures in excess of $1 \mathrm{keV}$ (and the ions required for lasing at soft-x-ray wavelengths are necessarily relatively heavy). Additionally, the presence of even relatively slight homogeneous broadening due to electron-ion collisions appears to be extremely deleterious to rebroadening due to the dominance of the associated Lorentzian distribution in the wings of the profile [16]. To reach the ultrashort, ultraintense regime using plasma-based amplifiers, therefore, requires collisional broadening (we do not consider isotopic broadening [17]). Unfortunately, there is a limit to how far conditions within soft-x-ray lasing media can be tailored to maximize collisional broadening because gain is destroyed through collisional deexcitation of the upper lasing level at high electron densities.

Nevertheless, seeding makes it possible (and is the only way) to overcome gain reduction due to increasing the linewidth of the amplifying transition to reach the ultrashort regime. Seed injection also allows the spatiotemporal characteristics of the plasma amplifier to be more fully exploited. 


\section{MODELING}

The rate of change of intensity of radiation propagating unidirectionally through plasma gain media can be expressed as $[10,18]$

$$
\begin{aligned}
\frac{d I(v, z)}{d z}= & \frac{j_{0}}{V\left(v_{0}\right)}\left[1+\frac{g_{0} I(v, z)}{j_{0}}\right] \\
& \times \int_{0}^{\infty} \frac{S(u) \varphi(v, u) d u}{1+\left(1 / I_{s}\right) \int_{0}^{\infty} I(v) \varphi(v, u) d v},
\end{aligned}
$$

where $I(v, z)$ is the intensity at frequency $v$ at a distance $z$ into the medium and $I_{s}, j_{0}$, and $g_{0}$ are the saturation intensity, emissivity, and small-signal gain coefficient at line center, respectively. $S(u)$ is the value of the inhomogeneous component of the profile at frequency $u$ and $\phi(v, u)$ is the value of the homogeneous component of the profile (both profiles being area normalized) centered on $u$ at $v$

$$
S(u)=\frac{1}{\Delta v_{G}}\left(\frac{4 \ln 2}{\pi}\right)^{1 / 2} \exp \left[\frac{-4 \ln 2\left(u-v_{0}\right)^{2}}{\Delta v_{G}^{2}}\right],
$$

and

$$
\phi(v, u)=\frac{2}{\pi \Delta v_{L}\left\{1+\left[4(v-u)^{2}(v-u)^{2} / \Delta v_{L}^{2}\right]\right\}} .
$$

Here, $v_{G}$ is the inhomogeneous full width at half maximum (FWHM) and $\Delta v_{L}$ is the homogeneous FWHM, $V\left(v_{0}\right)$ is the Voigt profile resulting from the convolution of independent homogeneous and inhomogeneous broadening mechanisms evaluated at the central frequency. It is given by

$$
V\left(v_{0}\right)=\int_{0}^{\infty} S(u) \phi\left(v_{0}, u\right) d u \text {. }
$$

Equation ( 1 ) is valid for plasma conditions that are independent of time, which is the case when the transit time of the x-ray laser through the plasma is shorter than hydrodynamic time scales. It is also valid for amplifiers pumped by traveling waves, as used in modern transient schemes to minimize energy requirements [19], provided the local plasma conditions "seen" by the plasma do not change as it progresses through the medium.

To benchmark our gain bandwidth calculations, we refer to work by Koch et al. [10] making use of a quasi-steady-state pumping scheme and measuring the intensity and spectral width of a $20.6 \mathrm{~nm}$ neonlike Se laser pulse as a function of distance into the plasma amplifier. Although this work was performed quite some time ago, to the best of our knowledge it remains the only test case with which linewidth predictions can be compared. We use Eq. (1) to model the radiative transfer process and make comparisons with the results obtained experimentally by Koch et al. For the plasma of their experiment, they determined the electron density to be $\sim 4 \times 10^{20} \mathrm{~cm}^{-3}$, the electron temperature to be $\sim 900 \mathrm{eV}$, and the ion temperature to be $\sim 400 \mathrm{eV}$. Under these conditions, the Gaussian component of line broadening due to Doppler broadening is $36 \mathrm{~mA}$.

A full quantum-mechanical treatment of collisional line broadening is formidable [20,21]. It requires not only cross sections for excitation and deexcitation out of the upper and lower levels defining the lasing transition to every other possible level, but quantum mechanical probability amplitudes for elastic scattering from both the upper and lower states of the transition into every solid angle [2I]. Instead, we make use of the impact approximation originally proposed by Lorentz [22] (which considers each collision with the radiating ion to result in a complete disruption of the wave train and loss of phase) and equate the FWHM in angular frequency units to twice the electron-ion collision frequency $[13,23]$. This approximation agrees with quantum mechanics if assumptions are made that are reasonably justifiable under conditions suitable for and when considering XRL operation [20]. It is applicable when the time between electron-ion collisions $\tau_{e-i}$ greatly exceeds the collision duration $t_{d}$, so that the target is effectively interacting with one perturber only at any one time

$$
\tau_{e-i}=\frac{1}{v_{e-i}} \gg t_{d}
$$

Here, $v_{e-i}$ is the electron-ion collision frequency [23]. Accordingly, this is valid for most electron-ion collisions. A rough estimate for $t_{d}$ can be obtained by dividing the distance between ions by the mean electron speed (i.e., by assuming that a collision is continuing to take place throughout the time the electron remains within the volume of the ion sphere). This approach, however, neglects the long-range character of the Coulomb force. An alternative reasonable definition is the Debye length $\lambda_{D}$ divided by the electron speed

$$
t_{d}=\frac{\lambda_{D}}{\left|v_{e}\right|} \simeq\left(\frac{\varepsilon_{0} m_{e}}{n_{e} e^{2}}\right)^{\frac{1}{2}}
$$

Here, $v_{e}$ is the electron velocity, $\varepsilon_{0}$ is the vacuum electrical permittivity, $m_{e}$ is the electron mass, $n_{e}$ is the electron density, and $e$ is the electronic charge. A more rigorous estimation is not necessary as it is generally safe to assume ions cannot be influenced at all by electrons farther away than a Debye length (certainly at the sort of temperatures and densities of relevance to this work). Therefore, any inaccuracies introduced by using the simple expression of $\mathrm{Eq}$. (6) can only result in an overestimation of the collision duration and so cause us to err on the side of caution when determining the validity of the impact approximation using Eq. (5). Electron temperature has no direct bearing on the collision duration when defined in terms of the Debye length in this way as it cancels.

\section{RESULTS}

A Lorentzian linewidth of $23.5 \mathrm{~mA}$ is predicted using this treatment under the conditions of Koch ef al. [10]. Koch et al. obtained values for $g_{0}, j_{0}$, and $I_{s}$ iteratively until a best fit to a plot of intensity versus distance using Eq. (1) was identified. They were estimated to be $5.5 \mathrm{~cm}^{-1}, 1.5 \times 10^{-9} \mathrm{~W}$ $\mathrm{cm}^{-3} \mathrm{~Hz}^{-1}$, and $1.7 \times 10^{-4} \mathrm{~W} \mathrm{~cm}^{-2} \mathrm{~Hz}^{-1}$, respectively. In Fig. 1, we plot our FWHM predictions with $36 \mathrm{~m} \AA$ Gaussian and $23.5 \mathrm{~m} \AA$ Lorentzian line broadening components as a function of distance into the plasma for the conditions of Koch et al. along with their experimentally obtained values. Also plotted are predictions for a much reduced Lorentzian component of $1 \mathrm{~m} \AA$ (again, with a Doppler component of $36 \mathrm{~m} \AA$ ), showing extensive saturation rebroadening. A Lorentzian component as small as this, however, is unrealizable for solid target laser-produced plasma amplifiers and no account is taken 


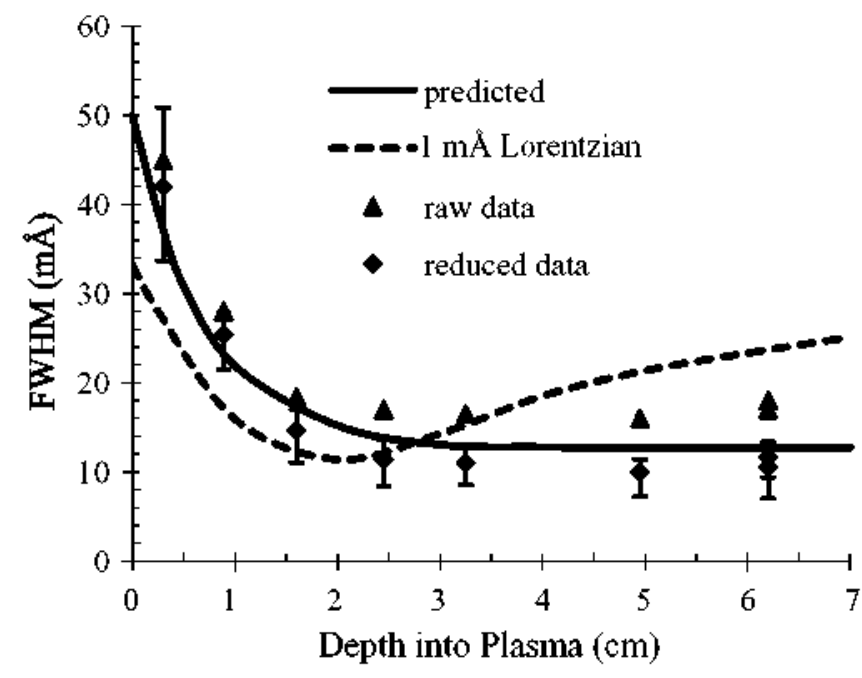

FIG. 1. FWHM determined experimentally by Koch et al. [10] and our predictions as a function of distance into the plasma amplifier with $36 \mathrm{~m} \AA$ of Doppler broadening and a $23.5 \mathrm{~m} \AA$ Lorentzian component, as calculated in this work (solid line). An unrealistic Lorentzian value of $1 \mathrm{~m} \hat{A}$ (dashed line) was also used to illustrate saturation rebroadening, though these predictions take no account of collisional redistribution (see main text).

of collisional redistribution of velocity. Raw and reduced experimental data points are plotted as originally presented. We note that, although the transit time through this plasma is $\sim 200 \mathrm{ps}$, and this is long compared to typical time scales for hydrodynamic evolution, agreement with experiment is generally very good. In particular, the resulting intrinsic linewidth of $50 \mathrm{~mA}$ agrees with that obtained by Koch et al. through extrapolation of the reduced data to $0 \mathrm{~cm}$. This affords confidence in our theoretical model.

Under the conditions of the Koch et al. experiment without seeding [10], the resulting amplified beam has a spectrally integrated intensity of $\sim 5.0 \times 10^{8} \mathrm{~W} \mathrm{~cm}^{-2}$ and the spectral bandwidth has narrowed to $\sim 13 \mathrm{~m} \AA$ after propagating 3 $\mathrm{cm}$ into the amplifier. It is noteworthy that for a Fourierlimited beam, this bandwidth corresponds to an unprecedented sub-500-fs duration. However, streak cameras measured an emission duration of $\sim 250$ ps [10], consistent with the long duration of the heating pulse. This confirms that significant gain persisted throughout the heating period and that the $x$-ray laser pulse resulted from many trains of amplification.

A study of the effects of electron density and temperature on spectral linewidth indicates that $100 \mathrm{fs}$ and shorter duration pulses are attainable with optimized but realistic plasma parameters-for instance, twice the electron density and half the electron temperature of the Koch et al. experiment [10] (i.e., $8 \times 10^{20} \mathrm{~cm}^{-3}$ and $450 \mathrm{eV}$, respectively). We estimate $g_{0}$ and $I_{s}$ under these revised conditions to be $\sim 60 \%$ of and $\sim 4.5$ times the original values, respectively. (We did not re-estimate $j_{0}$ as the seeding levels presented in Figs. 2 and 3 are so great as to render it insignificant.) These values are obtained by constructing simple rate equations to estimate the new level populations assuming steady-state conditions (as also assumed by Koch et al.). The collisional rates required for this for optically allowed transitions are estimated using oscillator

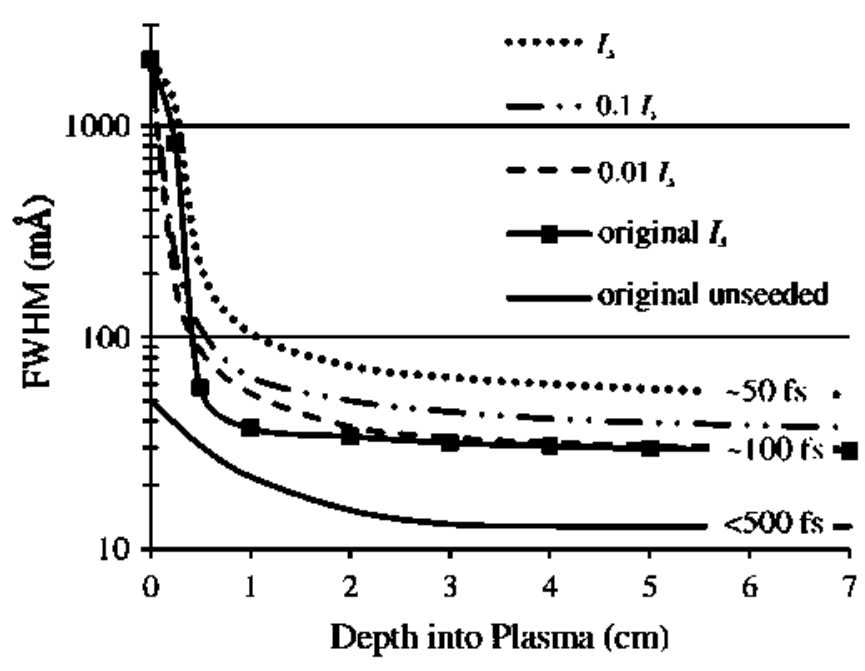

FIG. 2. (Color online) Predicted FWHM of a 20.6 nm laser pulse versus distance into an Ne-like Se plasma under differing plasma conditions. The unbroken lines show predictions under the original Koch conditions (see text) and the broken lines show those under the revised conditions (again, see text). In each case, the seeding level is given in terms of the respective saturation intensity, $I_{s}$

strengths obtained from the spontaneous decay rates given by Koch et al. and van Regemorter's formula [24]

$$
R_{l \rightarrow U}=3.2 \times 10^{-7}\left(\frac{E_{H}}{k_{B} T_{e}}\right)^{\frac{3}{2}} f_{l \rightarrow U} \frac{\exp (-y)}{y} G(y) .
$$

Equation (7) gives the rate of collisional excitation between levels $R_{l \rightarrow H}$, in units of $\mathrm{cm}^{3} \mathrm{~s}^{-1}$. Here, $E_{H}$ is the first ionisation potential of $\mathbf{H}, k_{B}$ is the Boltzmann constant, $f_{l \rightarrow u}$ is the oscillator strength for the transition concerned, and $y$ is the ratio of the transition energy to the electron temperature $T_{e}$. A limiting value of 0.2 for the Gaunt factor $G(v)$ for transitions between different quantum shells when $v>1$ was proposed [24]. This was used to obtain the rates between the ground state $\left(2 p^{6}\right)$ and the lower lasing level $\left(2 p^{5} 3 s^{1}\right)$. For transitions within the same quantum shell, a limiting value of 0.8 was used as this was found by later work to give reasonable results [25].

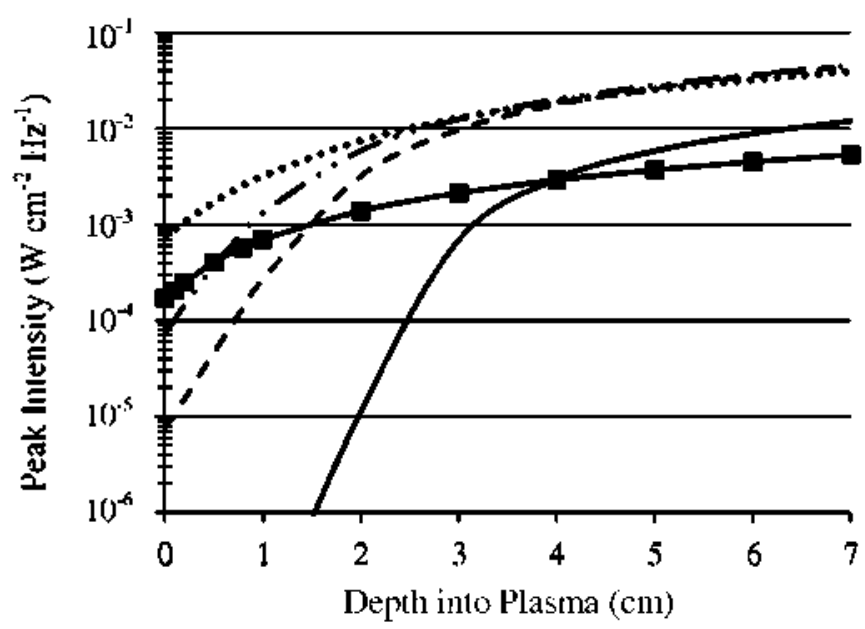

FIG. 3. (Color online) Peak intensities corresponding to Fig. 2 (same legend). 
For the lasing transition between the $3 p$ and $3 s$ subshells at $450 \mathrm{eV}, y=0.13$ and a value of 0.45 was obtained in this case by interpolation. Work showed that the van Regemorter formula is reasonably accurate for transitions within the same quantum shell [24], but there is doubt about its accuracy for transitions between shells [25]. In the present case, however, the only transitions between different shells for which it is used are collisional excitation and deexcitation between the lower lasing level and the ground state. As the rate of radiative decay to the ground state from the lower lasing level, given by the Einstein $A$ coefficient, dominates collisional rates between these two levels (as is required for lasing), any resulting loss of accuracy should not be too significant. In support of this, we note that the rate of radiative decay to the ground state from the lower lasing level is almost 5000 times greater than the collisional rate under the original conditions of Koch et al. [10], yet we are only considering a doubling of the electron density and halving of the electron temperature. Under the revised conditions, the rate of radiative decay to the ground state is $\sim 1800$ times greater than the collisional deexcitation rate. This was calculated by first recalculating the excitation rate by, in turn, recalculating the product of the excitation rate coefficient and ion density, multiplying this by 2 to account for the doubled electron density, and then applying the principle of detailed balance to obtain the inverse rate [24]. [Note that there is a typographic error in Eq. (4.6.2) of Ref. [24] used to apply the principle of detailed balance as there should be no minus sign in the exponent.] For the dipole-forbidden transition between the ground state and upper lasing level, the excitation and deexcitation rates under these revised conditions were obtained using the Eissner-Seaton formula [26] (with the statistical weight of the lower level-i.e., the ground state-equal to 1)

$$
R_{l \rightarrow u}=\frac{8.63 \times 10^{-6}}{\sqrt{T_{e}}} \exp (-y) Y_{l \rightarrow u} .
$$

Here, $Y_{I \rightarrow u}$ is a measure of the collision strength, $T_{e}$ is in kelvin, and, once again, this expression gives the rate in $\mathrm{cm}^{3}$ $\mathrm{s}^{-1}$. A value for $Y_{l \rightarrow u}$ was obtained by equating the excitation rate given by Koch et al. for this forbidden transition to that obtained using Eq. (8) under the original conditions. The collisional broadening contribution to linewidth under the revised conditions is predicted to be $\sim 50 \mathrm{~mA}$ and the time between collisions is over two orders of magnitude greater than the collision duration as defined by Eq. (6).

The resulting pulse linewidth and peak intensity predictions are shown in Figs. 2 and 3, respectively, under both the original Koch et al. conditions [10] and our revised conditions of twice the electron density and half the electron temperature for a range of seeding levels. We first consider the revised conditions with a seed intensity of $0.01 I_{s}\left(=7.5 \times 10^{-6} \mathrm{~W}\right.$ $\mathrm{cm}^{-2} \mathrm{~Hz}^{-1}$ ). This is equivalent to an integrated intensity of $1.2 \times 10^{9} \mathrm{~W} \mathrm{~cm}^{-2}$ and to an energy of $\sim 0.1 \mathrm{~nJ}$ in a $10 \mathrm{fs}$ pulse of $30 \mu \mathrm{m}$ diameter. The pulse shape as a function of time for this scenario is shown in Fig. 4. The spectral FWHM of the pulse has narrowed to $\sim 30 \mathrm{~m} \AA$ after traveling through the plasma and the corresponding Fourier-limited duration is $\sim 100$ ts; the pulse intensity increases by over two orders of magnitude to $\sim 1.6 \times 10^{11} \mathrm{~W} \mathrm{~cm}^{-2}$ and on focusing this beam
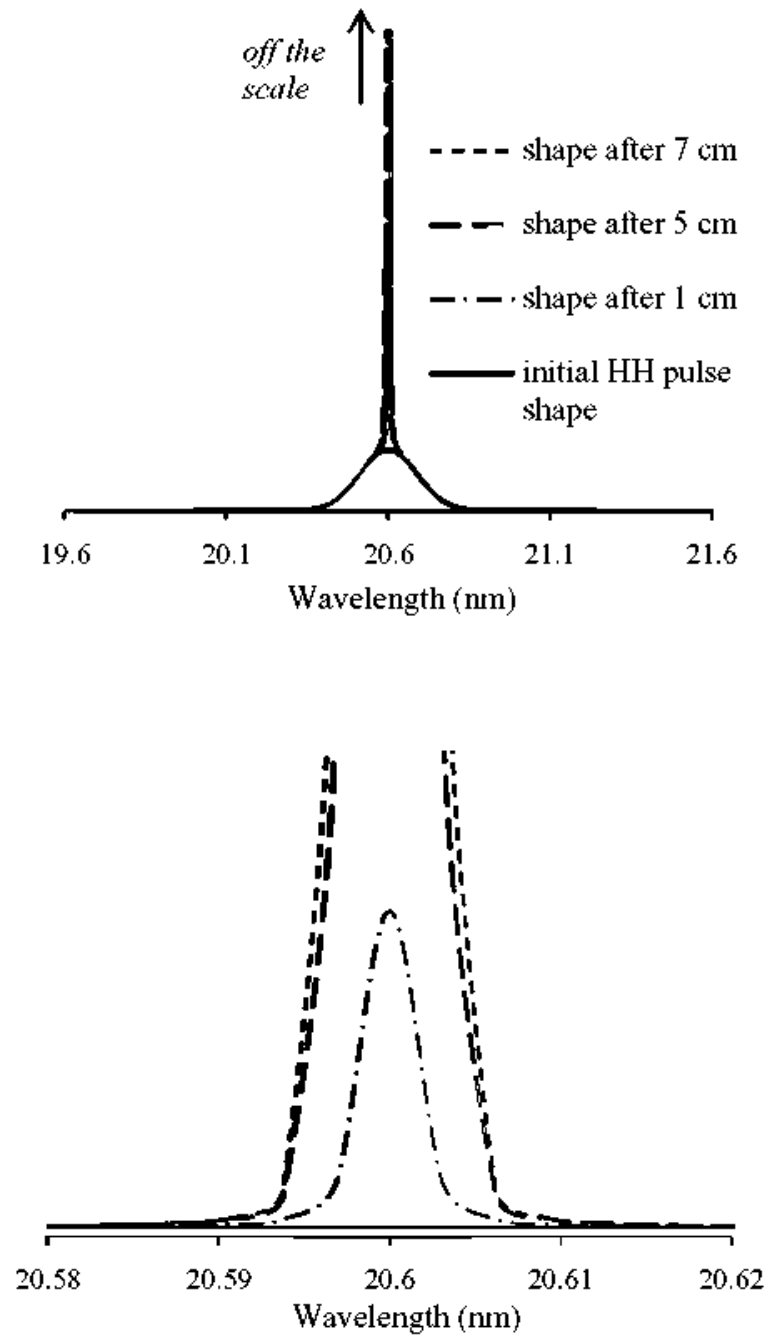

FIG. 4. (Color online) Top: Pulse shape as it passes through the plasma amplifier under the revised conditions with a seeding level of $0.01 I_{s}$ (see main text). Bottom: As above but with an expanded horizontal scale.

down to $0.1 \mu \mathrm{m}$, as discussed by Zeitoun et al., an intensity in excess of $10^{16} \mathrm{~W} \mathrm{~cm}^{-2}$ results.

Simulations using the EHYBRID $1.5 \mathrm{D}$ atomic physics and hydrodynamic code [27] show that the revised conditions are obtained over a lateral extent of $\sim 30 \mu \mathrm{m}$ at $\sim 400 \mathrm{ps}$ after the peak intensity of double-sided irradiation of a $0.15-\mu \mathrm{m}$-thick Se foil by long wavelength $(1.315 \mu \mathrm{m})$ pulses of $\sim 250$ ps FWHM and $\sim 2 \times 10^{13} \mathrm{~W} \mathrm{~cm}^{-2}$ intensity. This is shown in Fig. 5. Using half this pumping pulse intensity, a very similar electron density and temperature profile is predicted over the same lateral extent $\sim 250 \mathrm{ps}$ after peak long wavelength irradiation (of the same wavelength and, again, double-sided) of a 3 -mm-wide Se foil of $0.05 \mu \mathrm{m}$ thickness with 150 ps FWHM duration pulses. (Uniformity and temperature increase as foil thickness decreases.) As in the Koch et al. experiment [10], this second scenario requires $\mathrm{kJ}$ pumping $(\sim 6 \mathrm{~kJ})$, but a final intensity in excess of $10^{16} \mathrm{~W} \mathrm{~cm}^{-2}$ is predicted on focusing less stringently an initial $0.01 I_{s}$ seed down to $1 \mu \mathrm{m}^{2}$ and the energy of the output pulse is predicted to be of the order of tens of $\mu \mathrm{J}$. It is likely, however, that 


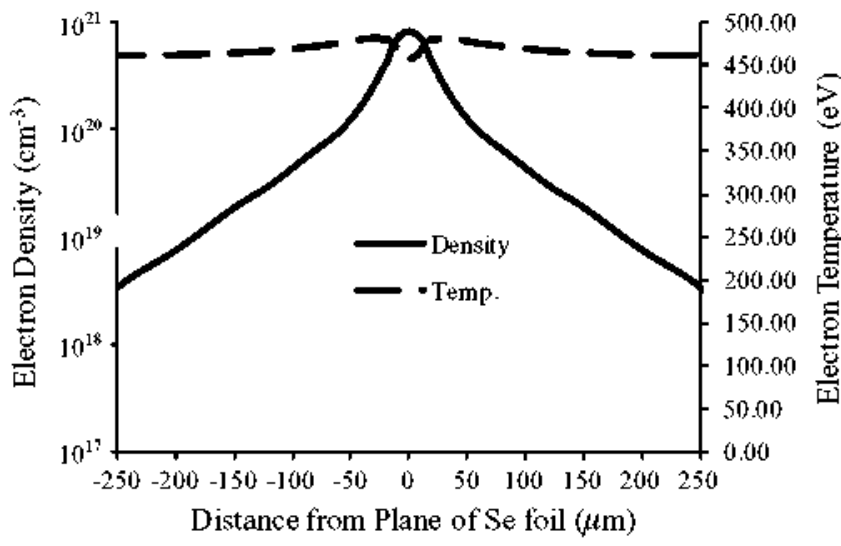

FIG. 5. (Color online) Plots of predicted electron density and temperature as a function of the distance from the longitudinal plane of an Se foil of $0.15 \mu \mathrm{m}$ initial thickness at $400 \mathrm{ps}$ after the peak intensity of double-sided irradiation by long wavelength pulses of 250 ps FWHM and $\sim 2 \times 10^{13} \mathrm{~W} \mathrm{~cm}^{-2}$ intensity. The simulation was performed using the EHYBRID $1.5 \mathrm{D}$ atomic physics and hydrodynamic fluid code [27].

the energy required to form a region of given volume where such desired conditions exist could be reduced significantly using transient, multiple pumping schemes, especially with Ni-like plasma amplifiers. To maximize validity, though, it was necessary to consider the same quasi-steady-state laser system used by Koch et al. The hydrodynamic conditions evolve faster than the transit time of the pulse through the (7-cm-long) plasma, so the pumping pulse wavefront would have to be angled accordingly. This initial seed intensity over a $3 \mathrm{~mm} \times 30 \mu \mathrm{m}$ area equates to $10 \mathrm{~nJ}$ in a $10 \mathrm{fs}$ pulse. We note that Zeitoun et al. [7] inferred a seed energy of $35 \mathrm{~nJ}$ from their experiment and much greater seed energies have been reported in the literature [28].

It is expected that the uniformity of the gain region could be increased significantly by tamping the Se target on both sides (by $\mathrm{CH}$, for instance) [29]. With a seed pulse of $0.1 I_{s}$ intensity under the revised conditions, sub-100-fs pulses ( $\sim 80 \mathrm{fs}$ ) of slightly higher intensity $\left(\sim 2 \times 10^{11} \mathrm{~W} \mathrm{~cm}^{-2}\right.$ for the unfocussed beam) are predicted. There is little point in seeding at much greater intensities than this, however, when gain is as low as this and when the bandwidth of the seed so greatly exceeds that of the amplifying transition as the increase in spectrally integrated intensity with plasma length becomes minimal.

We also note that, under our revised conditions, Eq. (1) (which considers intensity only) is expected to predict the same results as the much more involved and less physically intuitive density matrix equations [30] (which take account of electric field phase and amplitude also). This is because, although we are considering very short pulses, the relevant time scale is still long compared to the inverse of the Rabi frequency [30] $\Omega$ for the electric fields we consider here. Additionally, the collisional (and Doppler) components of the amplifying transition linewidth exceed (greatly) the natural component [31]. Furthermore, as Loudon states [31], the effective equivalence between the rate and density matrix equations is "most easily studied" in the limit of low intensity when $\Omega^{2} \propto I \ll \gamma \gamma_{\mathrm{sp}}$, where $\gamma$ is the total line broadening and $\gamma_{\mathrm{sp}}$ is the natural component due to spontaneous emission. For the example we give of seeding at $0.01 I_{s}$ under the revised conditions, because of the extent of collisional broadening, this condition is also satisfied using the results of Safronova et al. [32]. The ultrahigh intensities result from focusing down to close to the diffraction limit after the seeded pulse has exited the plasma amplifier.

\section{CONCLUSIONS AND DISCUSSION}

Ultrashort pulses can be obtained over a single gain lifetime with sufficiently strong seeding. If, as in the case of the Koch et al. experiment [10] using a quasi-steady-state regime, the upper lasing level is being repeatedly repumped and the plasma amplifier is long, the overall output would consist of a relatively intense, short-lived pulse superimposed on a weaker, longer background due to amplified spontaneous emission (ASE). This weaker, longer background could, in principle, be overcome by the choice of pumping scheme. If the plasma amplifier is sufficiently short that conditions are steady over the time scale taken for traversal by the seeded pulse, a transient scheme with normal pumping could be used to limit ASE due to repumping. If not, a transient scheme with traveling wave pumping could be used (with the pumping wave approximately coincident with the progression of the seeded pulse) so that the plasma conditions in the reference frame of the pulse remain the same. In each case, a sufficiently intense pulse would effectively "bleach" the plasma as it progresses, depleting the gain in its wake.

Relying on collisional as well as Doppler broadening to produce ultrashort pulses is unorthodox, but it is predicated on results indicating that the plasma still functions as an amplifying medium under conditions more suited to boosting gain bandwidth than maximizing gain. HH seeding results in a pulse with a very large initial bandwidth, and more of this bandwidth is preserved (so the pulse duration decreases in the Fourier limit) as the seeding level is increased. The gain inferred by Koch et al. [10] of $5.5 \mathrm{~cm}^{-1}$ is much less than that achievable routinely today [19], and we conclude that ultraintense (above $10^{16} \mathrm{~W} \mathrm{~cm}^{-2}$ ), ultrashort (less than 100 fs) pulses could be obtained using this technique with much shorter plasma amplifiers.

Support from TUIXS NEST-Adventure 012843 and SFINX-LASERLAB is gratefully acknowledged.
[1] G. S. Edwards, R. S. Austin, and F. E. Carroll, Rev. Sci. Instrum. 74, 3207 (2003).

[2] K. Tiedtke. A. Azima. N. von Bargen. L. Bittner, S. Bonfigt, S. Dusterer, B. Faatz, U. Fruhling, M. Gensch, Ch Gerth,
N. Guerassimova, U. Hahn, T. Hans, M. Hesse, K. Honkavaar, U. Jastrow, P. Juranic, S. Kapitzki, B. Keitel, T. Kracht, M. Kuhlmann. W. B. Li, M. Martins. T. Nunez, E. Plonjes, H. Redlin, E. L. Saldin, E. A. Schneidmiller. J. R. Schneider, 
S. Schreiber. N. Stojanovic. F. Tavella, S. Toleikis, R. Treusch. H. Weigelt, M. Wellhofer. H. Wabnitz, M. V. Yurkov, and J. Feldhaus, New J. Phys. 11. 023029 (2009).

[3] A. Barty, S. Boutet, M. J. Bogan, S. Hau-Riege, S. Marchesini, K. Sokolowski-Tinten, N. Stojanovic, R. Tobey, H. Elurke, A. Cavalleri, S. Düsterer, M. Frank, S. Bajt, B. W. Woods, M. Marvin Seibert, J. Hajdu, R. Treusch, and H. N. Chapman, Nat. Photonics 2, 415 (2008).

[4] S. Marchesini. S. Boutet. A. E. Sakdinawat, M. J. Bogan, S. Bajt. A. Barty. H. N. Chapman. M. Frank. S. P. Hau-Riege, A. Szöke. C. Cui. D. A. Shapiro. M. R. Howells, J. C. H. Spence, J. W. Shaevitz, J. Y. Lee, J. Hajdu, and M. M. Seibert. Nat. Photonics 2. 560 (2008).

[5] T. Mocek, B. Rus, M. Kozlová, J. Polan, P. Homer, K. Jakubczak. M. Stupka, D. Snopek, J. Nejdl, M. H. Edwards, D. S. Whittaker, G. J. Tallents, P. Mistry, G. J. Pert, N. Booth, Z. Zhai. M. Fajardo. P. Zeitoun. J. Chalupský. V. Hájková. and L. Juha. Eur. Phys. J. D 54. 439 (2009).

[6] A. Klisnick, J. Kuba, D. Ros, R. Smith, G. Jamelot, C. ChenaisPopovics, R. Keenan, S. J. Topping, C. L. S. Lewis, F. Strati, G. J. Tallents, D. Neely, R. Clarke, J. Collier, A. G. MacPhee, F. Bortolotto, P. V. Nickles, and K. A. Janulewicz, Phys. Rev. A 65. 033810 (2002).

[7] Pl. Zeitoun, G. Faivre. S. Sebban. T. Mocek. A. Hallou. M. Fajardo. D. Aubert, Ph. Balcou, F. Burgy. D. Douillet. S. Kazamias, G. de Lachèze-Murel. T. Lefrou. S. le Pape. P. Mercère, H. Merdji. A. S. Morlens, J. P. Rousseau, and C. Valentin, Nature (London) 431, 426 (2004).

[8] Y. Wang, E. Granados, M. A. Larotonda, M. Berrill, B. M. Luther, D. Patel, C. S. Menoni, and J. J. Rocca, Phys. Rev. Lett. 97. 123901 (2006).

[9] Y. Wang. M. Berrill. F. Pedaci. M. M. Shakya. S. Gilbertson. Zenghu Chang, E. Granados, B. M. Luther. M. A. Larotonda. and J. J. Rocca. Phys. Rev. A 79, 023810 (2009).

[10] J. A. Koch. B. J. MacGowan, L. B. Da Silva, D. L. Matthews, J. H. Underwood. P. J. Batson, R. W. Lee. R. A. London, and S. Mrowka, Phys. Rev. A 50, 1877 (1994).

[11] R. Tommasini, F. Löwenthal, and J. E. Balmer, Phys. Rev. A 59, 1577 (1999).
[12] P. V. Nickles. V. N. Shlyaptsev. M. Kalachnikov, M. Schnürer. I. Will, and W. Sandner. Phys. Rev. Lett. 78, 2748 (1997).

[13] A. Comey. Atomic and Laser Spectroscopy (Oxford University Press, New York, 1977).

[14] P. W. Smith and T. Hänsch. Phys. Rev. Lett. 26, 740 (1971).

[15] D. H. Schwamb and S. R. Smith. Phys. Rev. A 21, 896 (1980).

[16] G. J. Pert. Phys. Rev. A 50, 4412 (1994).

[17] K. R. Nambiar. Lasers: Principles, Types and Applications. 1st ed. (New Age International. New Delhi, 2004).

[18] L. W. Casperson and A. Yariv, IEEE J. Quantum Electron. 8, 80 (1972)

[19] J. Dunn et al. Phys. Rev. Lett. 84, 4834 (2000).

[20] M. Baranger, Phys. Rev. 111, 481 (1958).

[21] M. Baranger, Plyys. Rev. 112, 855 (1958).

[22] H. A. Lorentz. Proc. Acad. Sci. Amsterdam 8. 591 (1906).

[23] T. P. Hughes, Plasmas and Laser Light (Adam Hilger, Bristol. 1975).

[24] D. Salzmann, Atomic Physics in Hot Plasmas (Oxford University Press, New York, 1998)

[25] D. H. Sampson and H. L. Zhang, Phys. Rev. A 45, 1556 (1992).

[26] M. J. Seaton, in Atomic and Molecular Processes, edited by D. R. Bates (Academic Press. New York, 1962). p. 374.

[27] G. J. Pert. J. Fluid Mech. 131. 401 (1983).

[28] B. W. J. McNeil, J. A. Clarke, D. J. Dunning, G. J. Hirst, H. L. Owen, N. R. Thompson, B. Sheehy, and P. H. Williams, New J. Phys. 9, $82(2007)$.

[29] M. H. Edwards, D. Whittaker, P. Mistry, N. Booth, G. J. Pert, G. J. Tallents. B. Rus. T. Mocek, M. Koslová. C. McKenna. A. Delserieys, C. L. S. Lewis, M. Notley. and D. Neely, Phys. Rev. Lett. 97. 035001 (2006).

[30] J. T. Verdeyen. Laser Electronics (Prentice Hall. New Jersey. 1995), p. 643

[31] R. Loudon, The Quantum Theory of Light (Oxford University Press, New York, 2000).

[32] U. I. Safronova, T. E. Cowan. and M. S. Safronova. J. Phys. B 38. $2741(2005)$. 